\title{
Transgression(s), déviance(s), norme(s) et limite(s)
}

Ce sixième volume paraît six ans après la création de Convergences francophones (CF) et se voit marqué par deux événements. Tout d'abord, un déménagement virtuel : la plateforme de la revue a été déplacée de l'université Mount Royal à l'université de l'Alberta pour des raisons de logistique informatique. Les numéros et les articles bénéficient maintenant d'un numéro DOI, ce qui donne une plus grande stabilité à notre contenu. La revue reste cependant attachée à Mount Royal. Ensuite, CF a satisfait aux exigences du répertoire DOAJ : l'indexation de CF permettra, nous l'espérons, d'augmenter sa visibilité à l'avenir. Si la publication d'une revue scientifique en ligne en libre accès a pu paraître transgressif au monde académique à une certaine époque, force est de constater que ces revues aussi se plient à des normes.

C'est précisément du double thème des normes et des transgressions dont il est question dans ce numéro qui rassemble des contributions dont certaines, revisitées et évaluées pour l'occasion, furent présentées lors d'une journée d'étude tenue à l'université Mount Royal le 26 avril 2018.

Dans le monde occidental et occidentalisé, de la Genèse aux polémiques suscitées par les dessins satiriques de Charlie Hebdo, en passant par l'exercice de la censure du pouvoir politique et religieux, jusqu'à l'émergence d'une littérature dite transgressive, la question de la transgression et de la déviance implique le point de vue d'une norme et de son autorité. A chaque fois que le lexique désigne la transgression ou la déviance, ce sont les limites du cadre socioculturel dans lequel nous évoluons qui se voient (re)posées. Dans le cas de la France, les exemples de scandales littéraires et artistiques ne manquent pas. Sans avoir à évoquer les turbulences des Lumières ${ }^{1}$, la réception des Fleurs du mal (1857) et de Madame Bovary (1857), les revendications des surréalistes, le choc cubiste, la parution de Sodome et Gomorrhe (1921) et de J'irai cracher sur vos tombes (1946), pour ne citer que quelques exemples, montrent ainsi la pérennité du transgressif et du normatif. Plus récemment, et du côté des littératures francophones, les noms de Calixthe Beyala et Léonora Miano surgissent dès lors qu'est posé le rapport des auteurs postcoloniaux face aux institutions littéraires occidentales : dans les deux cas, ces auteures, de manière différente, transgressent des normes littéraires mais aussi culturelles en intégrant à l'écrit les normes de l'oralité comme dans le cas de Beyala (Brezault).

Cette notion est également dynamique dans le sens où les transgressions d'hier ne sont plus nécessairement celles d'aujourd'hui et vice-versa. En France, personne n'irait maintenant interdire aux femmes de porter un pantalon alors que c'était le cas au XIXe siècle (loi du 17 novembre 1800 abrogée en 2013). Plus récemment, la loi sur le mariage pour tous a été perçue comme une transgression pour certains tandis qu'elle devenait norme pour en annuler une autre préalablement établie. Ces récentes avancées sociales se traduisent-elles dans les lettres et les arts ?

\footnotetext{
${ }^{1}$ Voir entre autres le numéro 89 de la revue Tangence, «L'invention de la normalité au siècle des Lumières » (2009).
}

Cet article est disponible sous la Licence d'attribution CC BY-SA 2.0 
Le récent succès du film 120 battements par minute de Robin Campillo, qui retrace la création d'Act-up Paris à la fin des années 80 et la lutte des militants face à l'indifférence ou au rejet de l'homosexualité par une certaine frange de la population française et du pouvoir politique (Pastorello), semble aller dans ce sens. On peut aussi se demander si, dans l'ère post \#metoo ou \#balancetonporc, la représentation des femmes dans les arts et les lettres réussit à échapper complètement à l'emprise des constructions patriarcales et occidentales. L'évocation du "féminisme blanc" fait ainsi violemment réagir les partisans d'un féminisme à prétention universelle en France tandis qu'au Québec le sujet rencontre l'adhésion d'un public peut-être plus éclairé ou du moins plus ouvert : la réception de la traduction de l'ouvrage classique de Patricia Hill Collins, La pensée féministe noire (1990, 2016 pour la traduction), qui paraît la même année que le collectif $L e$ sujet du féminisme est-il blanc ? Femmes racisées et recherche féministe, se distingue singulièrement $\mathrm{du}$ ton des critiques françaises rencontrées lors de recherches menées sur ce sujet par le biais d'un moteur de recherche bien connu de nos jours. Si la recherche reste bien sûr à être approfondie, il n'en reste pas moins là un beau sujet d'étude de réception et/ou de sociologie portant sur la norme et la transgression du féminisme dans deux cultures francophones.

C'est peut-être du côté du domaine de la traduction où s'affirme depuis plus longtemps la relation entre norme et transgression d'un point de vue interculturel. " Une "bonne" traduction doit toujours abuser », c'est une « capture captatrice, séductrice et transformatrice " qui obéit à un principe de " fidélité violente " (Derrida 114). Ces propos curieux voire alarmants et apparemment contradictoires de Derrida proposent une redéfinition de la nature de la traduction, bien loin des "belles infidèles" de Nicolas Perrot D'Ablancourt, ces traductions 'corrigeant' et 'améliorant' l'original afin de les rendre plaisantes aux yeux et aux oreilles du public cible. A la fois fidèle et abusive, violente et respectueuse, la traduction chez Derrida doit nécessairement commettre des abus (Lewis 225), brouiller les limites entre l'original et la traduction et devenir un point de contact, de friction et de réconciliation entre les langues et les cultures. La traduction chez Derrida ne respecte ni les normes de la langue et de la culture cible ni celles de la langue et de la culture source; elle devient à la fois un acte et une production bouleversant les systèmes linguistiques et culturels. Ce "tournant abusif" en traduction littéraire concerne également le domaine audiovisuel où Abé Mark Nornes prône un "soustitrage abusif" ("abusive subtitling"), c'est-à-dire un sous-titrage qui refuse de se faire oublier et attire l'attention du spectateur sur le lieu même de la traduction : choix de termes, refus de traduire certaines paroles, position ou police du texte nonconventionnelles sont autant de techniques testant les limites et les normes de l'acte traductif. Le sous-titrage abusif sort la traduction de l'ombre et force ainsi le spectateur à entrer en dialogue avec le texte source.

Ce dynamisme se retrouve également dans le fait que la transgression agit en même temps qu'elle montre. Et c'est peut-être là que se trouve sa force provocatrice (Sirinelli). Cette valeur performative se retrouve bien dans les textes et les champs discursifs dont il sera question dans ce numéro. Des représentations des femmes au XIXe siècle en France aux littératures francophones d'Algérie et de 
l'océan Indien (Mayotte) en passant par la traduction, la question des normes, des limites et des transgressions apparaît à la fois constitutive et productrice de textes.

Dans « La matrice ou le drageoir aux transgressions. Lectures croisées des pathologies féminines fin-de-siècle », Laure-Hélène Tron-Ymonet part de la métaphore huysmansienne du « drageoir » afin d'examiner la matrice féminine dans sa dualité d'objet désirant et désiré, devenu « topos d'une représentation de l'horreur » chez certains médecins et romanciers de la fin du XIXe. Elle inscrit tout d'abord le thème dans une longue tradition qui atteint son apogée à la fin du XIXe siècle: le sexe féminin y garde à la fois les marques de la passivité et de la transgression. La littérature, plus particulièrement le roman naturaliste, s'approprie le discours médical et ratifie " un nouveau paradigme herméneutique ». Par les exemples présentés, l'auteure montre un effet de porosité entre l'écriture médicale et l'écriture littéraire qui, toutes les deux, tentent de créer une " mythographie négative » de la femme sexuellement active. Tout d'abord, elle s'intéresse à trois transgressions sexuelles féminines peu analysées par les études littéraires: la nymphomanie, le saphisme et l'onanisme. Son attention se tourne ensuite vers les déviances cliniques du siècle, telles l'hystérectomie et l'ovariotomie. Liberté et soumission féminine sont alors confrontées. Finalement, les discours finiséculaires sur la femme, littéraires ou médicaux, servent à prouver la domination masculine.

Dans son article « Le transgressif comme pratique textuelle politique dans l'œuvre de Rachid Mimouni » Azouz Ali Ahmed procède à une relecture et un décryptage rigoureux de quelques oeuvres de Rachid Mimouni. A travers une approche résolument interdisciplinaire (empruntant aux théories littéraires, à la linguistique, à la sociologie, à l'anthropologie, ou encore à l'Histoire à l'époque contemporaine), l'auteur explore les modalités relatives à l'articulation du transgressif et du politique dans le contexte de la narration. L'analyse aborde les thèmes tabous de la sexualité et ses déviations, de l'immoralité des individus, de la perte des valeurs traditionnelles, de la corruption mafieuse, de l'oppression de la femme selon trois axes, le premier consacré au renouvellement et à l'avancée des théories et concepts « transgression » et « politiques » dans le champ de la recherche de différentes disciplines, le second proposant une analyse des ruptures à la fois esthétiques et thématiques des romans où se discerne une dislocation de la société qui y est représentée. Le troisième explore les différents éléments du discours mimounien convergeant dans le sens des fractures pour dire différentes transgressions associées au politique comme visant à bousculer les stéréotypes et les assignations identitaires dans le discours politique algérien de l'époque également dénoncées par les romans aux jeux de miroirs entre culture, Histoire, politique et société.

L'article de Linda Rasoamanana, "Mémoires d'outretabou : formes et enjeux de l'outrance dans Tonton! Rends-moi ma virginité... de Nassur Attoumani » fait également écho à quelques problématiques développées par Ali Ahmed. En effet, le roman d'Attoumani s'inscrit dans la littérature comorienne de témoignage et de dénonciation des vices sociaux, plus précisément ceux de l'inceste et de la pédophilie. Selon l'auteure, le roman se distingue toutefois des autres productions comoriennes (et mahoraises) par l'usage de l'obscénité à outrance. La violence de 
l'écriture qui se lit à travers « les distorsions structurelles et génériques » du texte, transpose le dérèglement de la famille. A travers une esthétique d'exagération, Attoumani contextualise et réactualise la violence et l'obscénité du récit pour dénoncer au même titre que le bourreau la complicité tacite de la société.

Dans son article " La traduction, entre transgression et limite ", Salah Basalamah s'attelle à une tâche ardue, celle de définir l'étude de la traduction et son devenir, ses limites et ses transgressions. La nature inter- voire transdisciplinaire de la traduction et de son étude, la traductologie, rend toute définition délicate. En effet, comme le démontre l'auteur, la traduction dépasse les cadres que l'on souhaiterait lui imposer et son étude est en constante évolution. La traductologie, discipline qui s'intéresse à l'étude de la traduction en tant que processus, pratique, produit et production, demeure résolument interdisciplinaire, se refusant les limites d'une approche particulière. Les différents "tournants" traductologiques (linguistique, culturel, sociologique, cognitif) font constamment évoluer les normes et les limites, et donc la nature de la traduction. Véritable paradoxe, la traduction se veut à la fois transgressive, faisant fi des frontières et préservant l'altérité du contenu source, et normative, proposant des approches ethnocentristes qui apprivoisent l'altérité et renforcent les frontières.

Enfin, la section Varia accueille l'article collectif « Les Slaves du sud au prisme des Lumières et du romantisme » de Jean-Jacques Tatin-Gourier, Jasmina Nikcěvić et Dragan Bojević. Les auteurs s'intéressent à l'impact culturel français dans ce qu'il était convenu d'appeler la péninsule balkanique entre 1750 et 1850 . De la réception des idées des Lumières et des œuvres littéraires de la fin du XVIIIe siècle au rôle joué par Charles Nodier dans la diffusion d'une image stéréotypée des anciennes Provinces illyriennes, en passant par l'évocation des traducteurs du Phanar à Istanbul, cet article replace en contexte les acteurs, tant humains que spatiaux, d'une médiation culturelle qui, si elle a contribué à la dynamique identitaire des Slaves du sud, aura fait l'objet d'une reconnaissance tardive en France.

Pour leur soutien, nous tenons aussi à remercier Christiane Achour, Mohamed Aït-Aarab, Faouzia Bendjelid, Pedro Catharina, Jorge Calderón, Lieven D'hulst, Amel Maafa, Alexis Nuselovici (Nouss), Cynthia Parfait-Volanosy, Lucie Robert et Alexandre Wenger. 


\section{Bibliographie}

Brezault, Éloïse. « Le Scandale comme stratégie d'exotisme chez Calixthe Beyala et Léonora Miano : Le Rôle de l'institution littéraire face aux auteurs post/coloniaux. " Contemporary French and Francophone Studies, vol. 22, no. 1, 2018, pp. 58-66,

Derrida, Jacques. « Le retrait de la métaphore. » $P O \& S I E$ n7, 1978, pp. 103-126.

Hamrouni, Naïma et Maillé, Chantal. Le sujet du féminisme est-il blanc ? Femmes racisées et recherche féministe. Montréal : Remue-Ménage, 2016.

Hill Collins, Patricia. La pensée féministe noire. Traduit de l'anglais par Diane Lamoureux. Montréal : Les éditions du Remue-ménage, 2016.

Lewis, Philip E. "The Measure of Translation Effects.” The Translation Studies Reader. $3^{\text {ème }}$ édition. Dirigé par Lawrence Venuti. Routledge, 2012. pp. 220-239.

Nornes, Abé Mark. Cinema Babel: Translating Global Cinema. University of Minnesota Press, 2017.

Pastorello, Thierry. « 120 battements par minute. » Cahiers d'histoire. Revue d'histoire critique, no. 137, 2018, pp. 183-188.

Sirinelli, Jean-François. « La norme et la transgression. Remarques sur la notion de provocation en histoire culturelle. »Vingtième Siècle. Revue d'histoire, vol. no 93, no. 1, 2007, pp. 7-14.

Tangence. «L'invention de la normalité au siècle des Lumières. » Dirigé par Frédéric Charbonneau. no. 89, 2019, pp. 1-94.

\section{Image}

Leeyonce. « Academy of Fine Arts. » Pixabay, 24 décembre 2015, https://pixabay.com/photos/academy-of-fine-arts-black-and-white1551370/. Consulté le 23 décembre 2019. 